\title{
Effect of Edge-Functionalization on the Ease of Graphene Nanoribbon Aggregation in Solvent
}

\author{
Jonathan D. Saathoffa ${ }^{a}$ Paulette Clancy ${ }^{\mathrm{a}, *}$ \\ ${ }^{a}$ School of Chemical and Biomolecular Engineering, Cornell University, Ithaca NY 14853
}

\begin{abstract}
Edge-terminating oligomers are frequently used to aid in the dispersion of solutionprocessed, "bottom-up" graphene nanoribbons (GNRs). However, the choice of edge-termination is typically made in a trial-and-error process. In this study, Molecular Dynamics was used to contrast the aggregational tendencies of GNRs with a simple hydrogen edge-termination to those with polyethylene glycol (PEG) and $n$-alkoxy chains substituted along the GNR edges. Unlike similar previous work on graphene sheets, this study focuses the effects on aggregation of adding chains to the side of the GNR that are commonly used in bottom-up synthesis routes. We used Thermodynamic Integration to determine energetic barriers to face-to-face and sliding pre-determined aggregational paths. In contrast, Steered Molecular Dynamics simulations offered an unbiased window into GNR aggregation, allowing us to assess to what extent the real aggregational mechanism might be a combination of those two paths. We found that the sensitivity of the potential of mean force to different edge-terminations was highly dependent on the aggregational path chosen. Along the lowest energy barrier path (GNRs sliding together), PEG edge-terminations increased the barrier to aggregation, whereas
\end{abstract}

\footnotetext{
*Corresponding author. Tel: 606 255-8656. E-mail PQC1@ cornell.edu (Paulette Clancy)
} 
$n$-alkoxy chains not only decreased the energy barrier significantly, but also increased the range at which GNRs begin to attract each other.

\section{Introduction}

There has been considerable recent interest in fabricating Graphene Nanoribbons (GNRs) from a "bottom-up" synthetic route[1, 2, 3, 4, 5]. Unlike pristine graphene sheets, sufficiently narrow GNRs have a finite band gap due to nanoconfinement and edge effects[6,7]. This band gap can be opened wide enough to be useful for logic devices[8, 9]. Unlike other approaches for producing GNRs, such as lithography[10,11] and opening carbon nanotubes[12, 13], a synthetic approach promises small GNR widths, resulting in larger band gaps, and more uniform edge geometries [14].

Synthesis of these GNRs typically begins with polymerizing large, polyaromatic units, producing bulky polyphenylene structures. Subsequently, a suitable oxidation reaction induces ring closure, resulting in the final GNR structure. GNR synthesis can be performed either in solution or through a surfacemediated approach[3]. In addition to small widths and uniform edge geometries, this method also permits regular edge functionalization. This has been shown to modify the electronic properties of GNRs[15], as well as their self-assembly on a substrate[16]. If the GNRs are synthesized in solution, edge functionalization is particularly useful to reduce aggregation of the final ribbons. This is critical because pristine graphene does not disperse well in many solvents [17]. Other

large polyaromatic molecules may need to be bonded to oligomers in order to keep them stably dispersed[18, 19, 20].

Adding oligomeric chains to the edges of GNRs, as well as other polyaro- 
matic molecules, has been reasonably successful at dispersing them, but the best oligomer/solvent combination is usually chosen through a trial-and-error approach. While this approach may ultimately be successful in producing a dispersed solution, it obfuscates the design of a dispersible system without numerous experimental trials, especially if dispersibility is combined with a desire to simultaneously tune electronic and self-assembly properties. Instead, understanding how edge-termination choices behave on a molecular level would have the advantage of making the design process less time-consuming and more rational. While this understanding would be difficult to obtain experimentally, this is an area where computational techniques, such as Molecular Dynamics (MD), can be a useful tool. This has been demonstrated before in similar systems. For example, Shih et $a l$. used MD to provide parameters to estimate the rates of aggregation for pristine graphene in several solvents[21]. In addition, it was demonstrated that the interaction between graphene's quadrupole moment and the solvent adds dispersive stability in solvents such as hexafluorobenzene[22]. MD was also used to show that suitable alkyl chains could be designed to disperse polyaromatic molecules in an $n$-alkane solvent[23].

In this study, we investigate how three different choices of oligomeric chains attached to GNRs affect their aggregation. While previous work, mentioned above, looked at graphene in various solvents, as well as functionalization of relatively small polyaromatic molecules, relatively little MD work has been done on GNRs $[21,24,25,22,23,26]$. One such example studied various chains as edge terminations on polyaromatic molecules, but only examined chains of similar chemistry (branched and linear alkyl chains) [23]. With this in mind, we chose to study the effect of various chains on GNRs in solvent. This added complication is neces- 
sary to understand how GNRs aggregate, rather than aggregation in graphene and smaller organic molecules, showing how processing choices affect this tendency.

In our previous work, we calculated enthalpies of aggregation for GNRs with many different edge terminations [27]. While this provided a description of the properties of different GNR edge terminations and their influence on the surrounding solution, these results do not directly relate to the process of aggregation itself. Indeed, the molecular mechanism by which aggregation occurs is very poorly understood. This is an important omission because, like graphene, solution-processed GNRs have a strong tendency to aggregate even in the presence of edge-termination groups that were expressly added to disperse the material $[3,28,29]$. To understand how different edge-terminations affect the rates of aggregation, potentials of mean force (PMFs), determined along the major paths taken by GNRs to come into contact with each other, will be particularly useful as they provide insight into the energetic barriers to aggregation. There is a lack in our current understanding of aggegation, from the major pathways responsible for aggregation to the effect of edge-termination on aggregation, that provided the motivation for this work. 
In this paper, we applied MD techniques to study how three different edgeterminations affect the dispersibility of GNRs in $n$-methyl-pyrrolidone (NMP), a solvent that disperses pristene graphene particularly well compared to other solvents (Figure 1) [17]. In these three types of GNRs, each with a different edge-termination, one out of four hydrogen-terminated sites was substituted with oligomers of (a) polyethylene glycol (PEG), (b) $n$-alkoxy chains of equal length, as well as (c) a GNR with purely hydrogen-terminated edges, to act as a control.

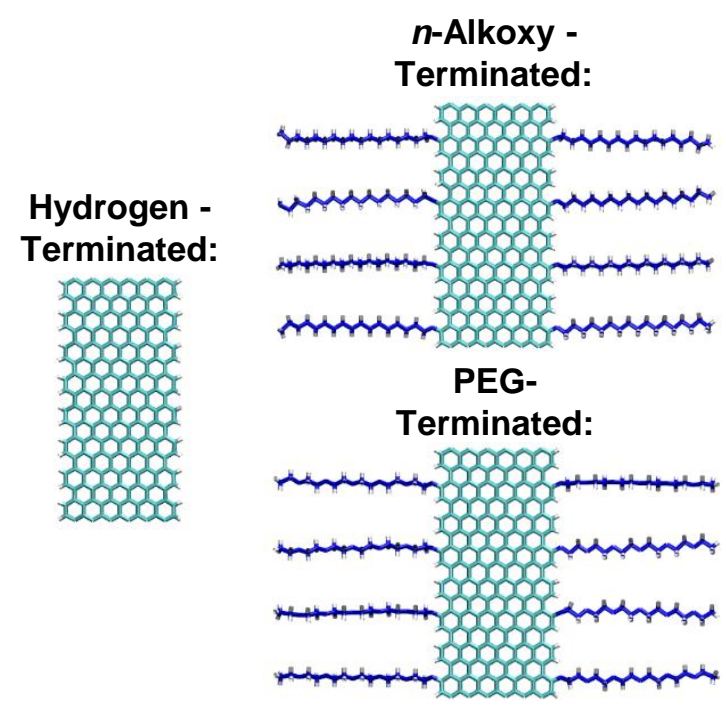

Figure 1: GNRs with the three edge-termination configurations used in this study. All hydrogen atoms are shown in white, graphene carbon atoms are shown in cyan, and other terminating chain atoms (carbon and oxygen) are shown in dark blue.

\section{Methods}

The LAMMPS package was used to run each MD simulation in this study [30]. In all cases, simulations were run with an NPT ensemble at 1 atm and $300 \mathrm{~K}$ 
with a 1 fs time step using a Nosé-Hoover thermostat and barostat. A 10 Å cut-off was applied to the van der Waals interactions with a tail correction. The particleparticle, particle-mesh method was used to model the long-range Coulomb interactions with a precision of $10^{-5}[31]$.

The OPLS-AA force field, with a few minor modifications, was used to represent the inter- and intra- molecular interactions that are the input needed for the MD simulations [32]. Since the polarity of the solvent has been shown to be important with respect to the stabilization of graphene in different solvents, partial charges on the solvent calculated using Density Functional Theory (DFT) were used as published by Aparicio et al. [33]. The Lennard-Jones interaction between the carbon atoms in graphene was altered to reproduce experimentally accurate graphene interlayer binding energies and separation distances $(\epsilon=0.0557$ $\mathrm{kcal} / \mathrm{mol} \sigma=3.4 \AA$ ) [21]. In addition, the dihedral terms for any four adjacent carbon atoms within the GNR were reparameterized to match the bending modulus of graphene, which was estimated through ab initio calculations [27, 34]. Additional information about the forcefield can be found in the Supplementary Information.

The GNRs used in this study are shown in Figure 1. The oxygen atoms in the $n$-alkoxy chains connect the $n$-alkane portion to the GNR. This is also true for the PEG chains, except that, in this case, there is an oxygen atom for every two carbon atoms. In each case, the chains have the same total combined number of carbon and oxygen atoms, namely 18. Both edge-terminated GNRs have the same grafting density (i.e., the number of chain terminations for every possible termination site). Both the length and grafting density were held constant in this study to focus attention on differences in chemistry between the two side-chains. However, 
it is expected that changes to these parameters would change the properties of the system [27]. GNRs with PEG-terminated chains with a similar grafting density to those shown in Figure 1 have been recently fabricated[28] .

Two different methods were used to calculate the Potential of Mean Force (PMF), or Free Energy, as a function of a reaction coordinate. The first is the Adaptive Biasing Force method (ABF) [35], and the second is the "Blue Moon" ensemble method or Thermodynamic Integration (TI) [36]. In each case, the PMF is calculated by numerically integrating the mean force along the reaction coordinate in the system:

$$
\frac{\partial F}{\partial x_{i}}=\left\langle\frac{\partial H}{\partial x}\right\rangle_{i}
$$

where $F$ is the free energy, $x$ is the relative position along the reaction coordinate, and $H$ is the Hamiltonian of the system.

In the $\mathrm{ABF}$ calculations, the system is initially permitted to move freely along its reaction coordinate. However, as the simulation progresses, the forces calculated along each point on the reaction coordinate are averaged, and the negative of the average force is imposed on the system, permitting the system to overcome free energy barriers. At the end of this simulation, these averaged forces are used to construct the final PMF. This method was used to calculate the adsorption free energy of PEG and $n$-alkoxy oligomers on a graphene sheet where free energy differences were expected to be small and the rate of diffusion was expected to be high with respect to significantly larger GNRs aggregating. More details on this procedure are in the Supplementary Information. 
Since ABF calculations in a simulation with GNRs aggregating would sample data too poorly to be useful, TI simulations were used to calculate the PMF of aggregation for graphene nanoribbons. In this method, separate simulations were set up along the reaction coordinate (the separation distance between neighboring GNRs), and the derivatives in Equation 1 were calculated in each one. The results were integrated using the trapezoidal rule to provide the final PMF. Two aggregational approaches were chosen for each GNR pair: face-to-face and sliding (Figure 2). In each case, the graphene's carbon atoms were held fixed unless they were located along the edges; these fixed atoms were designated as "core" atoms. While more degrees of freedom would be preferable, the slow dynamics of the system makes freely rotating and flexing GNRs prohibitively expensive to sample adequately. To obtain the configurations along the reaction coordinate, the GNRs were first drawn apart at a rate of $2 \AA / n s$ from the aggregated to dispersed state, and configurations were intermittently saved along the reaction coordinated to be used for the TI calculations. In addition, configurations were also obtained by drawing the GNRs together from a dispersed to aggregated state. The results from pulling the GNRs together and apart were compared to ensure that both systems were well equilibrated. More details on this procedure are in the Supplementary Information. 


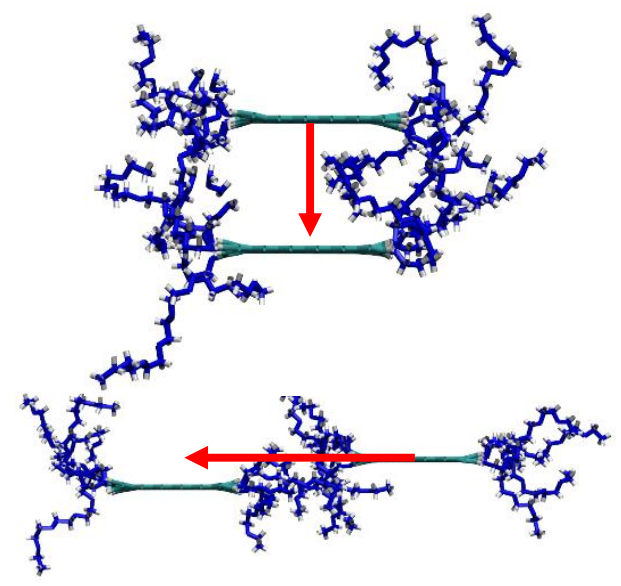

Figure 2: Cross-sectional views of GNRs approaching each other following a face-to-face path (top) and a sliding path (bottom). 
In order to initialize the TI runs, it was necessary to determine the equilibrium aggregated state (the relative positioning that the GNRs prefer to have when aggregated without constraints) so that the sliding and face-to-face trajectories could be directly compared with each other. This was achieved by running a simulation for $10 \mathrm{~ns}$ with aggregated, hydrogen-terminated GNRs. One GNR had its core atoms held fixed, while the other's core atoms were held rigid (by rigid, we mean that they were free to translate and rotate, but not bend or stretch relative to each other). The most probable offsets in the $x, y$, and $z$ directions were taken to be the equilibrated aggregation offsets for the GNRs to be used in subsequent TI simulations (see Figure 3). While this stacking pattern is consistent with Bernal stacking for pristine graphene, there is an offset of $1.3 \AA$ in the $x$ direction. This offset is due to the hydrogen-carbon dipoles along the edges lining up in a favorable fashion (negatively charged carbon atoms being attracted to positively charged hydrogen atoms in the neighboring GNR). 


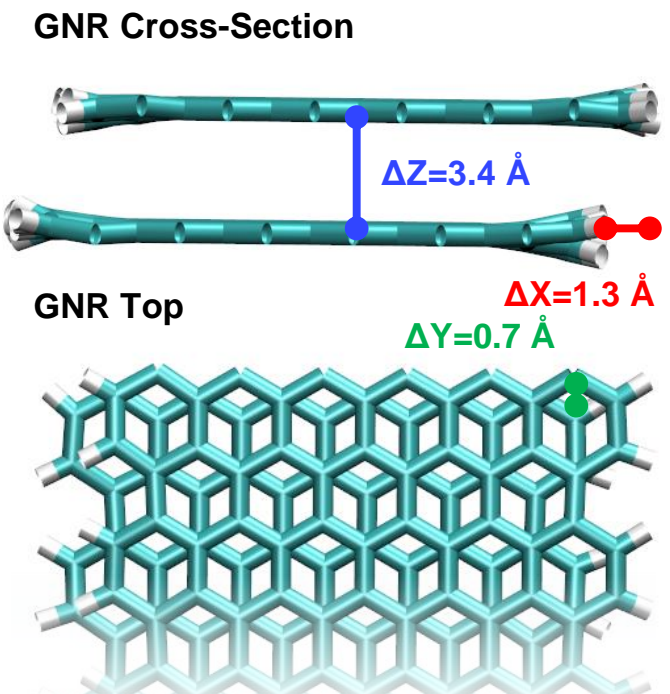

Figure 3: Cross-sectional (top) and top-view (bottom) illustrations of the offsets calculated for the equilibrated GNR aggregate in the $x, y$, and $z$ directions. 


\section{Results and Discussion}

\subsection{Face-to-face aggregational approach}

Figure 4 shows PMFs of the GNRs with the three different edge configurations for a face-to-face approach. This behavior is nearly identical to what has been observed for pristene graphene without edge termination [21]. Figure 5 shows several renderings of the hydrogen-terminated GNRs at different separations. At the farthest separation distance, the GNRs are separated by two layers of adsorbed NMP solvent. As the GNRs come together, one of the solvent layers is slowly forced out. This is responsible for the first peak, located between $10.8 \AA$ to $9.0 \AA$. The local minimum at $7.8 \AA$ represents the point where there is a single layer of solvent adsorbed to both GNRs. The large $(170 \mathrm{kcal} / \mathrm{mol})$ energy barrier at $6.4 \AA$ is the location at which the strongly adsorbed NMP is excluded and replaced with vacuum. Finally, the GNRs aggregate together at $3.4 \AA$ (note that this separation distance mirrors the value obtained when we determined the equilibrium interribbon distance, as shown in Figure 3. The hydrogen-terminated GNR has a large free energy of aggregation $(170 \mathrm{kcal} / \mathrm{mole})$ that is very close to that found in other studies[21]. However, since the magnitude of barriers dropped very significantly between the barrier at $6.4 \AA$ and $10.8 \AA$, it is unlikely that features farther away that $12.0 \AA$ would significantly change the calculated PMFs. 


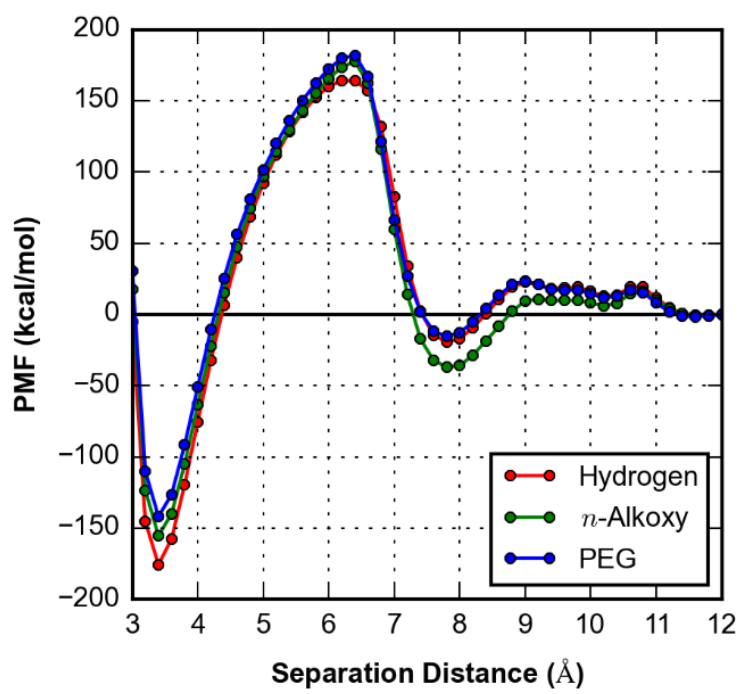

Figure 4: PMFs calculated for GNRs approaching in a face-to-face manner showing that all three edge-terminations produce essentially identical results. Color key as shown in the inset. 


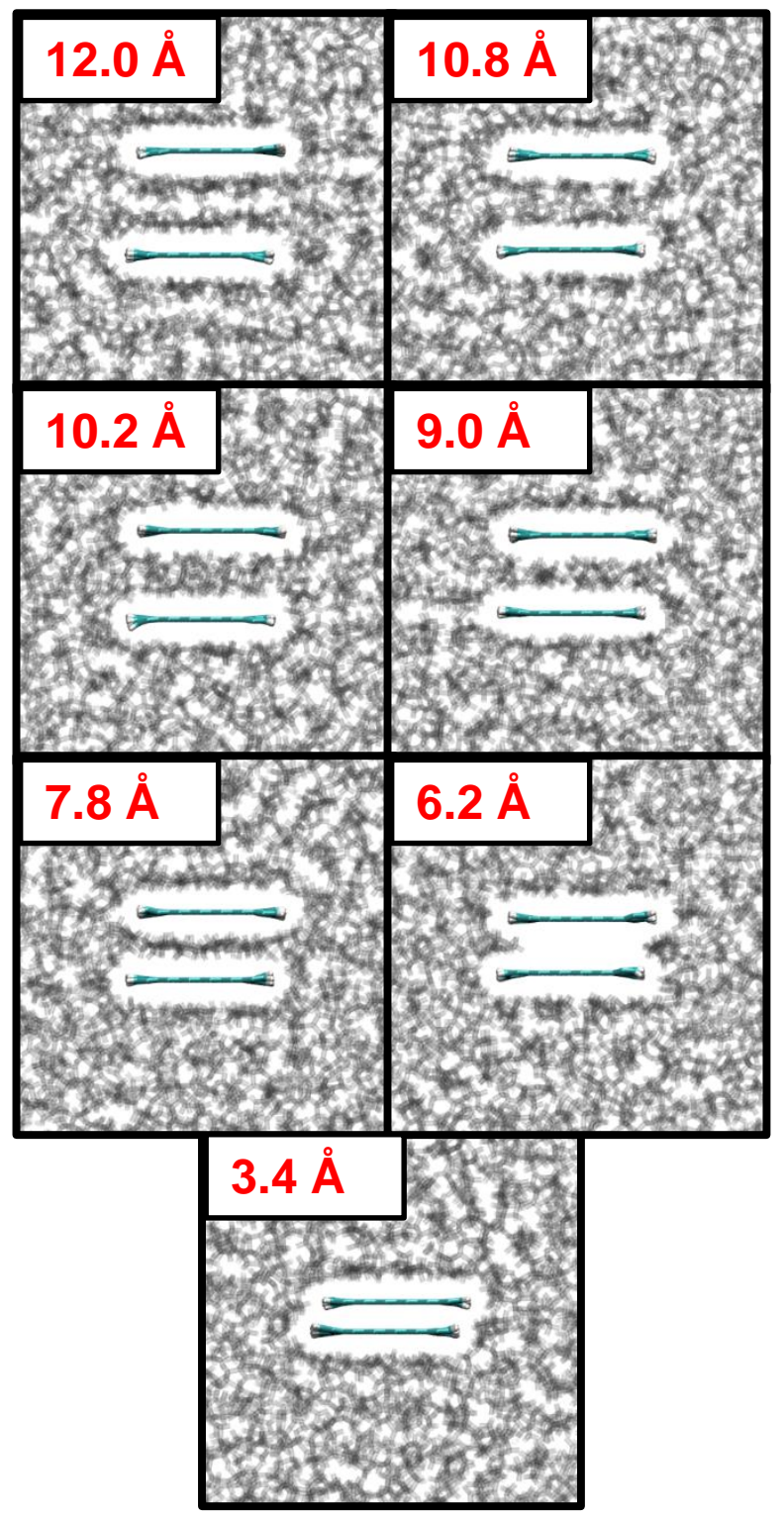

Figure 5: Cross-sectional views of hydrogen-terminated GNRs aggregating in a face-to-face manner. From top to bottom, these MD snapshots, representing different inter-ribbon separations (shown in red), illustrate how intervening solvent layers are excluded to form the fully aggregated state at a separation of $3.4 \AA$. 
The PMF curves presented in Figure 4 for the three different edge-terminations are very similar. This implies that edge terminations play a relatively small role in face-to-face aggregation. This similarity is evident in Figure 6, showing crosssections of $n$-alkoxy and PEG chains at a representative separation, here $7.8 \AA$. While individual chains periodically adsorb outside and inside the two GNRs during the aggregational process, most of the energetic contribution comes from the solvent and the faces of the GNRs themselves, and not the side-chains. It is important to notice the very large barrier in the PMF at $6.4 \AA$ : With a barrier of 164 $\mathrm{kcal} / \mathrm{mol}$ for $n$-alkoxy and PEG chains, we would not expect GNRs to ever come together along this (face-to-face) reaction path. However, in an unconstrained system, GNRs could flex or only come together in a face-to-face fashion over a small area. Even with a smaller area (a GNR only one ring long, for example), this value would scale to $14.3 \mathrm{kcal} / \mathrm{mol}$ - still a significant barrier. This will be discussed further in a later section. 


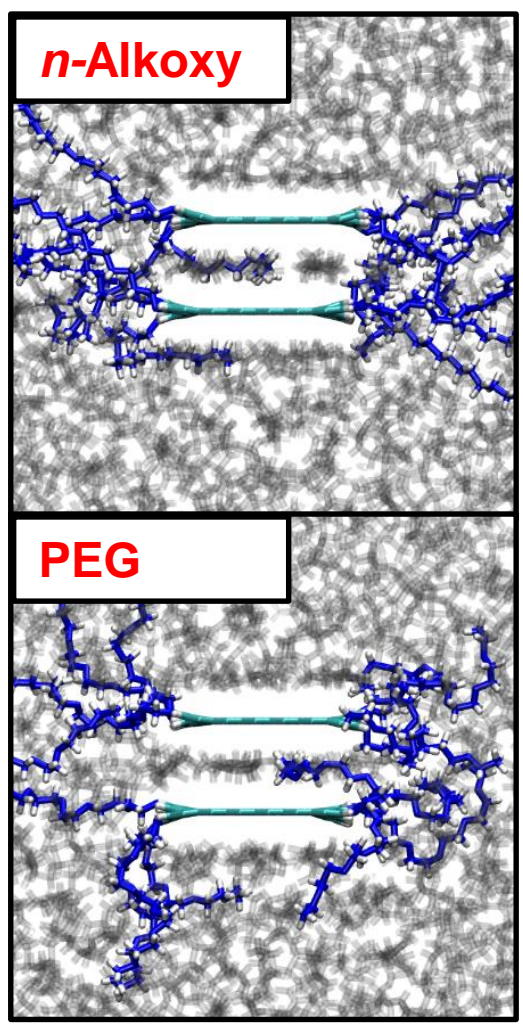

Figure 6: Cross-sectional images of GNRs with $n$-alkoxy (top) and PEG side-chains (bottom) approaching in a face-to-face manner at a $7.8 \AA$ A separation. 
In the sliding approach, the side terminations are more easily able to adsorb on the neighboring GNRs. To measure this interaction, we calculated the free energy of adsorption for the two oligomers on a graphene surface. $\mathrm{ABF}$ calculations were conducted for an $n$-alkane (to represent the $n$-alkoxy edge configurations - the oxygen atom was not included in these $\mathrm{ABF}$ calculations because it only serves to link the chain to the GNR) - and PEG oligomers on a sheet of graphene (Figure 7). We determined that the free energy of adsorption for a single PEG chain and $n$-alkoxy chain, each of which are seven non-hydrogen atoms long, are 0.9 $\mathrm{kcal} / \mathrm{mol}$ and $3.2 \mathrm{kcal} / \mathrm{mol}$, respectively. Clearly, $n$-alkoxy side-chains will adsorb much more strongly to GNR faces than would be the case for PEG side-chains adsorbing onto GNRs. While the free energies of adsorption on graphene will not be the same as the GNRs (the entropy change upon adsorption will almost certainly be different than this experiment using $\mathrm{ABF}$ because of the different constraints placed on the edge-termination chain on GNRs versus the isolated chain on graphene), the significant increase in adsorption energy for the $n$-alkane chain over the PEG chain shows that this effect will almost certainly be relevant for the GNR system. 

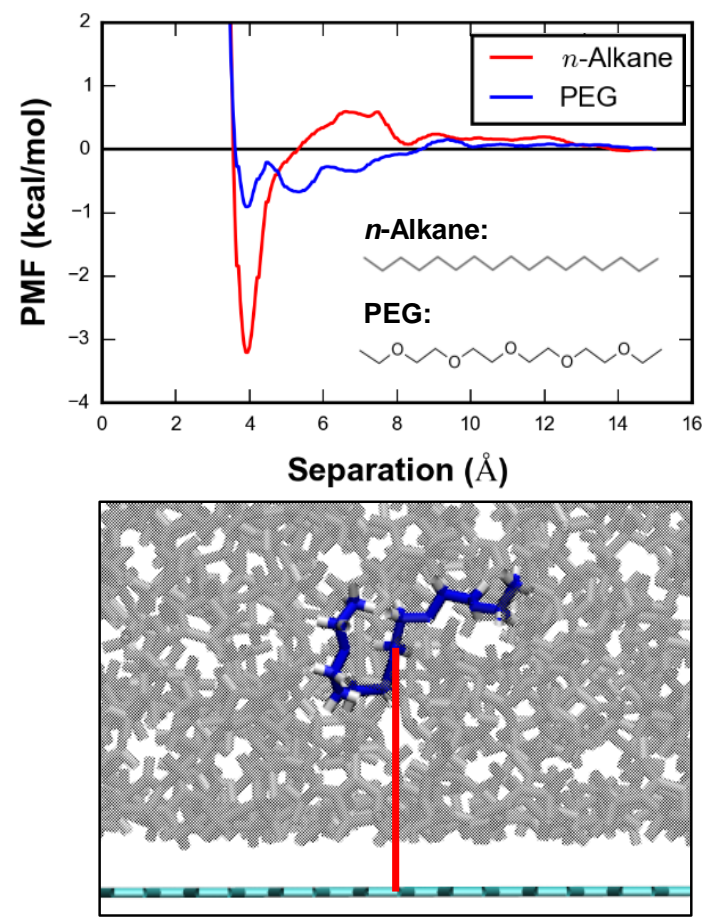

Figure 7: PMFs of $n$-alkane and PEG chains during adsorption on graphene in NMP (upper graph), and a representative side-view image of a PEG chain (dark blue) in NMP solvent (grey) above a graphene sheet (cyan) (lower image). While it may appear that there is a vacuum layer between the solvent and graphene, in reality, the height of the gap simply represents the sum of the van der Waals radii between the solvent and graphene. 


\subsection{Sliding aggregational approach}

Figure 8 shows the PMFs of the three different edge-terminated GNRs approaching in a sliding approach. In stark contrast to the face-to-face approach, the PMF of each edge-termination is qualitatively distinct, because now the sidechains of each edge-termination approach each other, rather than the faces of the GNRs. Starting with the hydrogen-terminated GNRs, Figure 9 shows crosssectional views at different separation distances. There are only very small fea-

tures in the PMFs of the GNRs until the edges are $23.0 \AA$ apart. At this point, there is a small minimum as only a single layer of solvent can pass between the GNRs. Next, a small maximum is reached at $19.0 \AA$ where that solvent layer is evacuated, and the PMF begins a steady decrease at increasingly smaller separation distances. This downward trend accelerates when the carbon atoms from neighboring GNRs begin to overlap. Finally, a fully aggregated state is reached at a separation of $1.0 \AA$, consistent with the results provided in Figure 3 for the $x$ offset. 


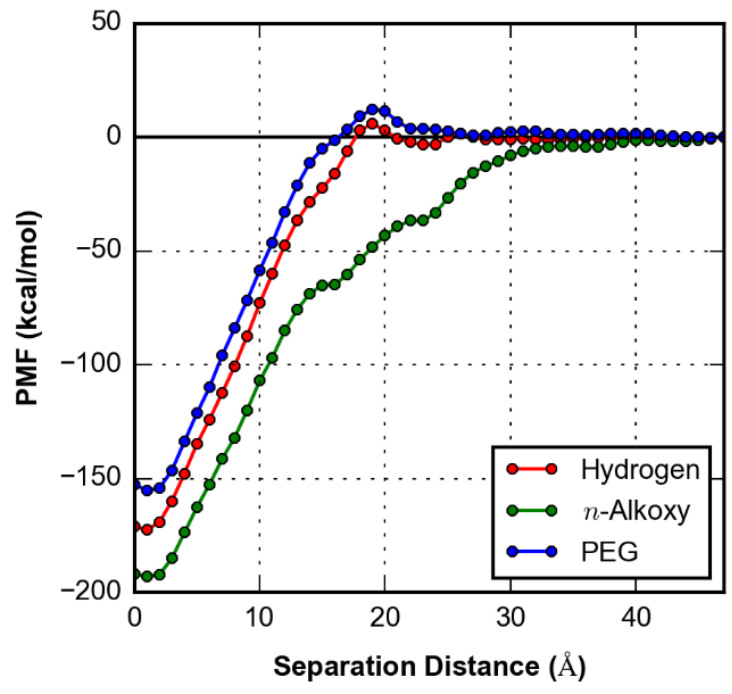

Figure 8: PMFs calculated for GNRs approaching in a sliding approach for the three different edge-terminations. Here, unlike Figure 5, the PMFs for n-alkoxy and PEG chains are significantly different. Color key as described in the inset. 


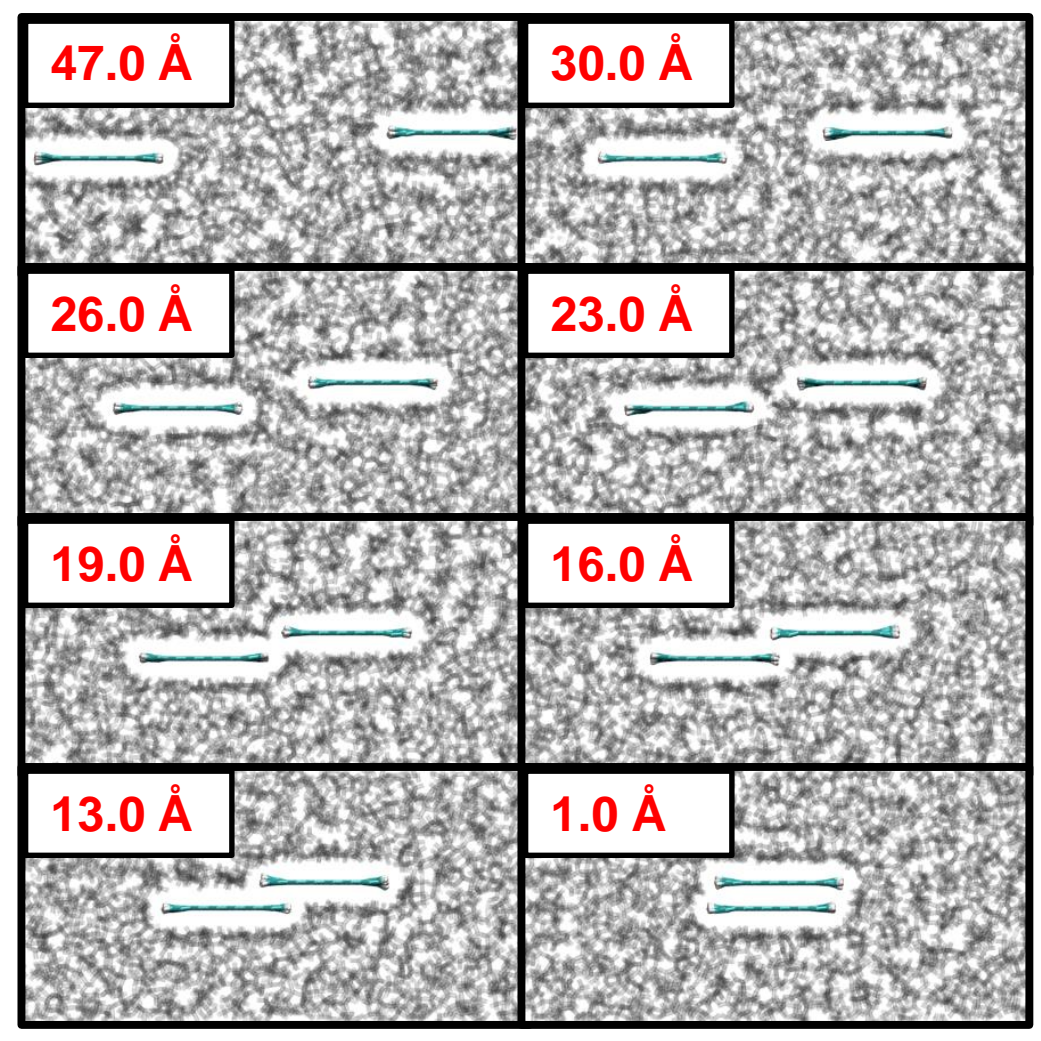

Figure 9: Cross-sectional views of the hydrogen-terminated GNRs aggregating in a sliding approach at different offsets (shown in red). In this case, there is no intervening solvent layer during aggregation. 
While the hydrogen-terminated behavior is very similar to that observed for pristine graphene [24], the two other GNR variants exhibit a very different behavior. Figure 10 shows cross-sectional views of the $n$-alkoxy-terminated GNR and the PEG-terminated GNR at a separation distance of $23 \AA$. At large and short separation distances, the PEG- and hydrogen- terminated GNRs display very similar features. However, this is not the case in the intervening distances between 13.0 $\AA$ and $23.0 \AA$ A. For the most part, the PEG chains distort the solvent between the GNRs, flattening out the features seen in the hydrogen-terminated GNRs. However, the peak is larger by $6.4 \mathrm{kcal} / \mathrm{mol}$, with a total height of $12.3 \mathrm{kcal} / \mathrm{mol}$, in the PMF at $19.0 \AA$ due to steric repulsion by the PEG chains.

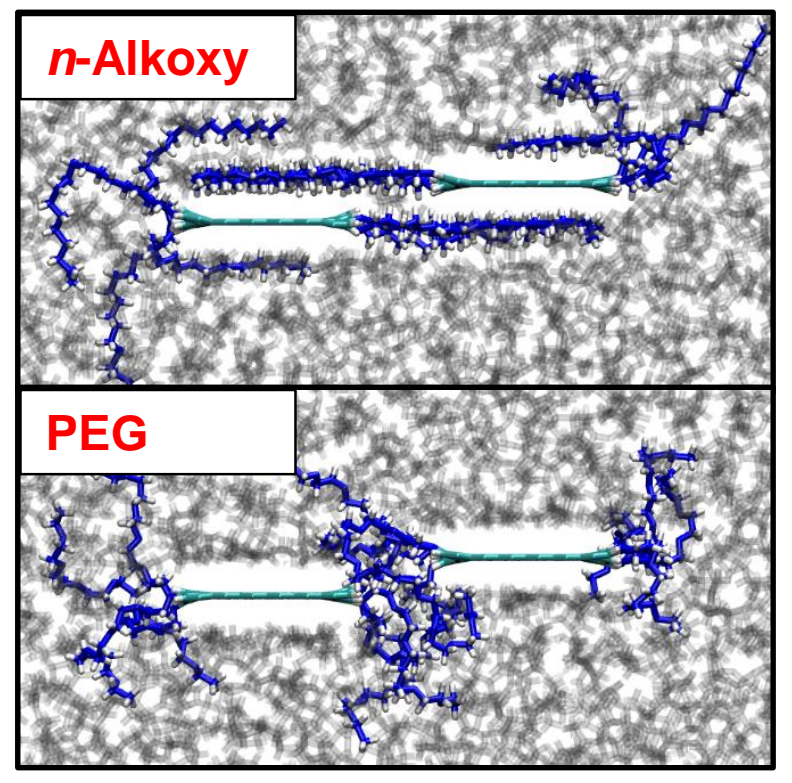

Figure 10: Cross-sectional images of $n$-alkoxy- (top) and PEG-terminated (bottom) GNRs approaching in a sliding approach captured at a $23.0 \AA$ offset, showing the extreme steric congestion of side-chains in the PEG case. 
In Figure 10, it is clear that this increase is due to steric congestion caused by the PEG side-chains. The case is quite different for the $n$-alkoxy-terminated GNR where no barrier is present. In fact, the PMF begins to decrease (i.e., increasing aggregational tendencies) at a much farther separation distance. From Figure 7, it is clear that the $n$-alkoxy chains are strongly adsorbed to the neighboring GNRs. This was same effect was described previously with the $n$-alkane chains being adsorbed on graphene sheets. Between $23 \AA$ and $13.0 \AA$, the main contributors to the PMF changes from interactions between the $n$-alkoxy chains (as they begin to slide off the opposite side of the neighboring GNR) to interactions between the two GNRs as they begin to come in contact with each other. At even closer distances, the PMF continues to decrease until it reaches its final, aggregated state.

The most significant difference between the $n$-alkoxy-terminated GNRs and the other two edge-terminations is the total absence of a barrier along the sliding aggregation path, whereas the hydrogen-terminated (albeit with an insignificant peak in the PMF) and PEG-terminated ones have peaks of $5.9 \mathrm{kcal} / \mathrm{mol}$ and $12.3 \mathrm{kcal} / \mathrm{mol}$, respectively. In addition, the $n$-alkoxy chains begin drawing the GNRs closer together at much larger separation distances that the other edge terminations. The small or non-existent barriers in the PMF for the various edgeterminated GNRs indicate that a sliding approach is a far more likely path for GNRs to follow than the face-to-face trajectory studied above.

\subsection{SMD Calculations}

In a more realistic aggregational process, the GNRs would be capable of many more degrees of freedom: They would be able to rotate freely with respect to each other, and they would be permitted to flex and bend. In order to demonstrate how fully flexible GNRs that are free to rotate might come together, two 
hydrogen-terminated GNRs starting at a face-to-face separation distance of $18.0 \AA$ (where the GNRs were not interacting with each other) were brought together using Steered Molecular Dynamics (SMD). In SMD, an artificial harmonic potential with a slowly changing equilibrium distance is added to the system. In this case, each end-point of the harmonic potential is placed on the center of mass of either GNR. The equilibrium separation distance was set to retract at a rate of $1 \AA / \mathrm{ns}$. Snapshots of this simulation are shown in Figure 11. At first, the GNRs come together oblivious of the presence of the other GNR (Figure 11.A and Figure 11.B). When they approach to a distance at which they are separated by just one solvent layer, the GNRs rotate with respect to each other (by roughly 90 degrees) so that the minimal amount of surface area approaches the other GNR (Figure 11.C). As the GNRs continue to be pulled together, they begin to warp in shape, billowing out from one another, and with a single layer of solvent remaining between the GNRs. The rotation and warping are clearly due to the fact that the energy barrier of the GNRs approaching in a full face-to-face manner would be too large for the GNRs to overcome, even with the reduced surface area that they have achieved (by rotation) in this simulation. As the GNRs continue their approach, the edges of the GNRs come in contact with one another (Figure 11.D). This small area of contact facilitates the exclusion of solvent between the GNRs, and the GNRs quickly adhere to each other along their entire length (Figure 11.E and Figure 11.F). While this SMD path may not represent all possible paths to aggregation, this kind of behavior was seen from multiple different starting points. The simulation was repeated with GNRs that had $n$-alkoxy- and PEG- edge-terminations with a retraction rate of $2 \AA / n s$. In all cases, the path taken was the same as that illustrated in Figure 11. This clearly shows that GNRs avoid approaching each 
other in a face-to-face mechanism, and instead come together at their edges.

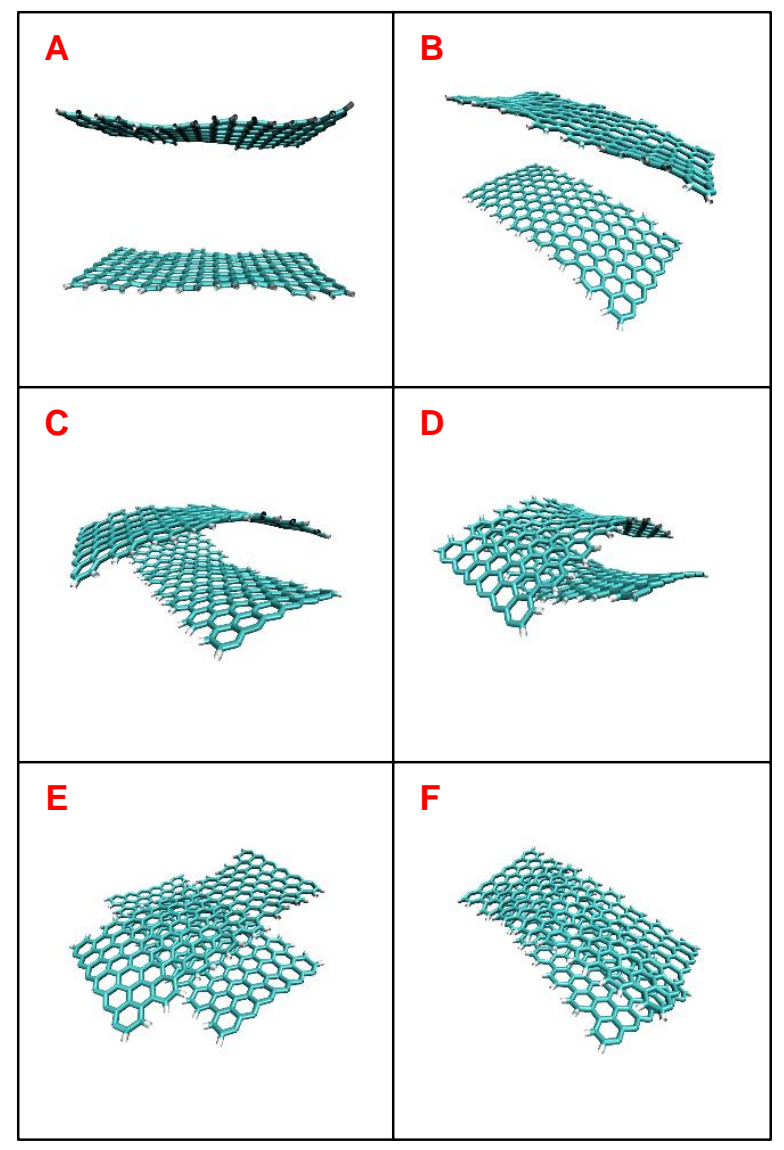

Figure 11: Representative images at different separation distances of two hydrogen-terminated GNRs approaching each other in a largely unconstrained manner as made possible by SMD. The separation distances are $18.0 \AA$ (A), $12.6 \AA$ (B), $5.9 \AA$ (C), $3.9 \AA$ (D), $3.7 \AA$ (E), and $2.7 \AA$ (F). The surrounding NMP solvent is not shown for clarity. 


\section{Conclusions}

In this paper, we have calculated the PMF of aggregating GNRs using TI along two pathways that essentially represent limiting and orthogonal strategies for aggregation, namely face-to-face and sliding trajectories. We found that a sliding trajectory is likely to be a far more energetically favorable aggregational pathway than a face-to-face approach, due to the much lower barriers displayed in the PMF by the former. We observed that the choice of edge-termination can significantly affect the barrier to aggegation along the sliding trajectory: PEG groups increased the barrier to aggregation relative to hydrogen-terminated GNRs by a significant amount, around $6.4 \mathrm{kcal} / \mathrm{mol}$. In contrast, $n$-alkoxy terminating groups actually remove the barrier to aggregation in the PMF and also increase the range of attraction between neighboring GNRs by about $10 \AA$. This shows that the addition of linear terminating groups such as these will only modestly improve the GNRs' aggregational tendencies or even increase the likelyhood of aggregation.

With its less accurate but far less constrained philosophy, SMD results validated the hypothesis that the large barrier to face-to-face aggregation will prevent GNRs from aggregating along that path. It is clear that approaching ribbons will distort by both rotation and bending to minimize their overlapping surface area. Instead, once they are close enough to touch at any point, this initiates solvent exclusion between the GNRs and aggregation is inevitable and rapid.

A major hindrance to these calculations was the computational cost. We found that, in order to obtain precise results for the PMFs, it took 24 ns runs that both pulled the GNRs apart and drew them together to avoid hysteresis of the curves. As an example of the cost, calculating the PMF for the $n$-alkoxy-terminated GNRs along the sliding trajectory illustrated in Figure 8 required a cumulative simula- 
tion time of $2.3 \mu \mathrm{s}$, or 2.3 billion time steps. In order to speed future calculations, smaller GNRs could be simulated by first comparing the results to these larger GNRs to see if they are qualitatively similar. If this could be done, additional trajectories could be attempted, such as edge-to-face calculations as well as using bulkier, branched edge-terminations. Increasing the throughput of these calculations would not only permit the comparison of many different edge terminations to understand why some perform better than others, but also allow these techniques to become a predictive tool through which to choose optimized edge-terminations that minimize GNR aggregational tendencies. Finally, while these simulations were performed exclusively for GNRs, they are likely to be similar for other twodimenesional materials.

\section{Acknowledgements}

The authors acknowledge the financial support of the National Science Foundation Nanoelectronics Beyond 2020 (NEB) program, award number CHE-1124754, and a gift from the NRI, award number (Gift \# 2011-NE-2205GB). We also acknowledge many helpful discussions with our NEB program collaborators, Profs. W. R. Dichtel (then in Chemistry and Chemical Biology, Cornell) and Prof. Y.L. Loo (Chemical and Biological Engineering, Princeton University). This work also benefitted from using the computational resources at the Extreme Science and Engineering Discovery Environment (XSEDE), which is supported by National Science Foundation grant number ACI-1053575 and by donations of high performance chips from the Intel Foundation. 


\section{References}

[1] Cai, J., Ruffieux, P., Jaafar, R., Bieri, M., Braun, T., Blankenburg, S. et al, Atomically precise bottom-up fabrication of graphene nanoribbons, Nature 466 (7305) (2010) 470-473. doi:10.1038/nature09211.

[2] Yang, X., Dou, X., Rouhanipour, A., Zhi, L., Räder, H.J. and Müllen, K., Two-dimensional graphene nanoribbons, J. Am. Chem. Soc. 130 (13) (2008) 4216-4217. doi:10.1021/ja710234t.

[3] Narita, A., Feng, X., Hernandez, Y., Jensen, S.A., Bonn, M., Yang, H. et al, Synthesis of structurally well-defined and liquid-phaseprocessable graphene nanoribbons, Nat. Chem. 6 (2) (2014) 126-132. doi:10.1038/nchem.1819.

[4] Vo, T.H., Shekhirev, M., Kunkel, D.A., Orange, F., Guinel, M.J.F., Enders, A. et al, Bottom-up solution synthesis of narrow nitrogen-doped graphene nanoribbons, Chem. Commun. 50 (32) (2014) 4172-4174. doi:10.1039/C4CC00885E.

[5] Vo, T.H., Shekhirev, M., Kunkel, D.A., Morton, M.D., Berglund, E., Kong, L. et al, Large-scale solution synthesis of narrow graphene nanoribbons, Nat. Commun. 5 (2014) 3189. doi:10.1038/ncomms4189.

[6] Barone, V., Hod, O. and Scuseria, G.E., Electronic structure and stability of semiconducting graphene nanoribbons, Nano Lett. 6 (12) (2006) 27482754. doi:10.1021/n10617033.

[7] Son, Y.W., Cohen, M.L. and Louie, S.G., Energy gaps in 
graphene nanoribbons, Phys. Rev. Lett. $97 \quad$ (2006) 216803. doi:10.1103/PhysRevLett.97.216803.

[8] Novoselov, K.S., Falko, V.I., Colombo, L., Gellert, P.R., Schwab, M.G. and Kim, K., A roadmap for graphene, Nature 490 (7419) (2012) 192-200. doi:10.1038/nature11458.

[9] Schwierz, F., Graphene transistors, Nat. Nanotechnol. 5 (7) (2010) 487-496. doi:10.1038/nnano.2010.89.

[10] Chen, Z., Lin, Y.M., Rooks, M.J. and Avouris, P., Graphene nano-ribbon electronics, Physica E 40 (2) (2007) 228-232. doi:10.1016/j.physe.2007.06.020.

[11] Han, M.Y., Özyilmaz, B., Zhang, Y. and Kim, P., Energy band-gap engineering of graphene nanoribbons, Phys. Rev. Lett. 98 (20) (2007) 206805. doi:10.1103/PhysRevLett.98.206805.

[12] Kosynkin, D.V., Higginbotham, A.L., Sinitskii, A., Lomeda, J.R., Dimiev, A., Price, B.K. et al, Longitudinal unzipping of carbon nanotubes to form graphene nanoribbons, Nature 458 (7240) (2009) 872-876. doi:10.1038/nature07872.

[13] Jiao, L., Zhang, L., Wang, X., Diankov, G. and Dai, H., Narrow graphene nanoribbons from carbon nanotubes, Nature 458 (7240) (2009) 877-880. doi:10.1038/nature07919.

[14] Hunter, J.D., Matplotlib: A 2d graphics environment, Computing In Science \& Engineering 9 (3) (2007) 90-95. doi:10.1109/MCSE.2007.55. 
[15] Jippo, H. and Ohfuchi, M., First-principles study of edge-modified armchair graphene nanoribbons, J. Appl. Phys. 113 (18) (2013) 183715. doi:10.1063/1.4804657.

[16] Kastler, M., Pisula, W., Wasserfallen, D., Pakula, T. and Müllen, K., Influence of alkyl substituents on the solution-and surface-organization of hexaperi-hexabenzocoronenes, J. Am. Chem. Soc. 127 (12) (2005) 4286-4296. doi:10.1021/ja0430696.

[17] Hernandez, Y., Nicolosi, V., Lotya, M., Blighe, F.M., Sun, Z., De, S. et al, High-yield production of graphene by liquid-phase exfoliation of graphite, Nature Nanotech. 3 (9) (2008) 563-568. doi:10.1038/nnano.2008.215.

[18] Yan, X., Li, B. and Li, L.S., Colloidal graphene quantum dots with well-defined structures, Acc. Chem. Res. 46 (10) (2012) 2254-2262. doi:10.1021/ar300137p.

[19] Xiao, S., Kang, S.J., Wu, Y., Ahn, S., Kim, J.B., Loo, Y.L. et al, Supersized contorted aromatics, Chem. Sci. 4 (5) (2013) 2018-2023.

[20] Ito, S., Wehmeier, M., Brand, J.D., Kübel, C., Epsch, R., Rabe, J.P. et al, Synthesis and self-assembly of functionalized hexaperi-hexabenzocoronenes, Chem. Eur. J. 6 (23) (2000) 4327-4342. doi:10.1002/1521-3765(20001201)6:23;4327::AID-CHEM4327¡3.0.CO;27.

[21] Shih, C.J., Lin, S., Strano, M.S. and Blankschtein, D., Understanding the stabilization of liquid-phase-exfoliated graphene in polar solvents: molecu- 
lar dynamics simulations and kinetic theory of colloid aggregation, J. Am. Chem. Soc. 132 (41) (2010) 14638-14648. doi:10.1021/ja1064284.

[22] Kocman, M., Pykal, M. and Jurečka, P., Electric quadrupole moment of graphene and its effect on intermolecular interactions, Phys. Chem. Chem. Phys. 16 (7) (2014) 3144-3152. doi:10.1039/C3CP54701A.

[23] Konatham, D. and Striolo, A., Molecular design of stable graphene nanosheets dispersions, Nano Lett. 8 (12) (2008) 4630-4641. doi:10.1021/nl802262p.

[24] Fu, C. and Yang, X., Molecular simulation of interfacial mechanics for solvent exfoliation of graphene from graphite, Carbon 55 (2013) 350-360. doi:10.1016/j.carbon.2012.12.083.

[25] Oyer, A.J., Carrillo, J.M.Y., Hire, C.C., Schniepp, H.C., Asandei, A.D., Dobrynin, A.V. et al, Stabilization of graphene sheets by a structured benzene/hexafluorobenzene mixed solvent, J. Am. Chem. Soc. 134 (11) (2012) 5018-5021. doi:10.1021/ja211225p.

[26] Azar, N.S. and Pourfath, M., Aggregation kinetics and stability mechanisms of pristine and oxidized nanocarbons in polar solvents, The Journal of Physical Chemistry C 120 (30) (2016) 16804-16814. doi:10.1021/acs.jpcc.6b05318.

[27] Saathoff, J.D. and Clancy, P., Simulation of graphene nanoribbon aggregation and its mediation by edge decoration, J. of Phys. Chem. B 119 (13) (2015) 4766-4776. doi:10.1021/jp510203j. 
[28] Gao, J., Uribe-Romo, F.J., Saathoff, J.D., Arslan, H., Crick, C.R., Hein, S.J. et al, Ambipolar transport in solution-synthesized graphene nanoribbons, ACS Nano 10 (4) (2016) 4847-4856.

[29] Huang, Y., Mai, Y., Beser, U., Teyssandier, J., Velpula, G., van Gorp, H. et al, Poly (ethylene oxide) functionalized graphene nanoribbons with excellent solution processability, J. Am. Chem. Soc. 138 10136-10139.

[30] Plimpton, S., Fast parallel algorithms for short-range molecular dynamics, J. Comput. Phys. 117 (1) (1995) 1-19. doi:10.1006/jcph.1995.1039.

[31] Hockney, R.W. and Eastwood, J.W., Computer simulation using particles, CRC Press, 1989.

[32] Jorgensen, W.L., Maxwell, D.S. and Tirado-Rives, J., Development and testing of the opls all-atom force field on conformational energetics and properties of organic liquids, J. Am. Chem. Soc. 118 (45) (1996) 11225-11236. doi:10.1021/ja9621760.

[33] Aparicio, S., Alcalde, R., Davila, M.J., Garcia, B. and Leal, J.M., Measurements and predictive models for the n-methyl-2pyrrolidone/water/methanol system, J. Phys. Chem. B 112 (36) (2008) 11361-11373. doi:10.1021/jp712131j.

[34] Kudin, K.N., Scuseria, G.E. and Yakobson, B.I., C 2 f, bn, and c nanoshell elasticity from ab initio computations, Phys. Rev. B 64 (23) (2001) 235406. doi:10.1103/PhysRevB.64.235406.

[35] Darve, E. and Pohorille, A., Calculating free energies using average force, J. Chem. Phys. 115 (20) (2001) 9169-9183. doi:10.1063/1.1410978. 
[36] Carter, E., Ciccotti, G., Hynes, J.T. and Kapral, R., Constrained reaction coordinate dynamics for the simulation of rare events, Chem. Phys. Lett. 156 (5) (1989) 472-477. doi:10.1016/S0009-2614(89)87314-2. 